\title{
Die Medizin in der Sicht eines italienischen Denkers der Aufklärung *
}

Von Loris Premuda

«Die positive Rolle der Aufklärung in der Medizin ist erstaunlich wenig bekannt», stellte E.H.Ackerknecht fest ${ }^{1}$. Vor ihm hatte P.Diepgen die Periode «von Leibniz bis zum Ausklang der Romantik» als «das Zeitalter starken Einflusses der Philosophie auf die Medizin» bezeichnet ${ }^{2}$. Diese beiden Stellungnahmen unterstreichen zwei Tatsachen: erstens, daß die Medizin der Aufklärungszeit nur ungenügend erforscht ist, zweitens, den starken Einfluß des philosophischen Denkens der Zeit von 1700-1830 auf die Medizin, die erst später in die zweite naturwissenschaftliche Periode eintrat. In diesem Zusammenhang ist es gerechtfertigt, auf einen lombardischen Schriftsteller der Aufklärungsepoche, den Philosophen, Wirtschaftler und Politiker Pietro Verri ${ }^{3}$ hinzuweisen und seine Äußerungen zur Medizin vorzustellen. Das folgende Zitat mag als Visitenkarte dieses kämpferischen Denkers gelten: «Es sind die Bücher der Philosophen, welche die Gerichte dazu zwangen, ihre starren Verfahrenstraditionen aufzugeben, nicht mehr gegen angebliche Hexen und Zauberer zu wüten, sie nicht mehr zu foltern, keine entsetzlichen Körperstrafen wegen Gesinnungsdelikten mehr zu verhängen und die Todesstrafe auf Ausnahmefälle zu beschränken. Dank den Philosophen haben die Bücher den Ehrenplatz erobert in einer Gedankenwelt, die vorher von den skrupellosen Verbreitern volkstümlicher Irrtümer und abergläubischer Vorstellungen beherrscht war. Wir verdanken es der Philosophie, wenn uns heute bei unsern Krankheiten vernünftige, aufgeklärte Ärzte und nicht mehr unkundige Scharlatane beistehen.» ${ }^{4}$ Und F.Diaz schreibt über ihn: «Tatsächlich vermochte Verri wie wenige in seinem Werk die Verbreitung der Ideen und das konkrete Handeln zu vereinigen: eine Fähigkeit der Verbindung von Wort und Tat, die für manche Persönlichkeiten charakteristisch ist, welche in Italien die Aufklärung und deren Reformideen verkörperten.» ${ }^{5}$

Unsere kurze Darstellung soll eine knappe Übersicht über die wichtigsten Werke Verris geben, soweit sie medizinische Themen betreffen: Lehrstoffe, Berufsethik, Studiengang, Methodologie. Verri schöpft aus verschiedenen

* Herrn Prof. Huldrych M.Koelbing zum 60. Geburtstag gewidmet. 
Iatrophysikern, aus Malpighi, Boerhaave und Morgagni. Er schreibt: «Die Medizin ist nichts anderes als Physik, auf den menschlichen Körper angewandt, also auf jene Maschine, die heute noch sehr mangelhaft bekannt ist und wahrscheinlich auch nie vollständig bekannt sein wird.» ${ }^{6}$ Der Unterschied zwischen der einen und der andern Krankheit ist größer als derjenige zwischen dem einen und dem andern gesunden Körper. Nie hat es vielleicht seit den Zeiten von Hippokrates «zwei völlig identische Krankheiten» gegeben ${ }^{7}$. Gerade in dieser Vielfalt ist die Unsicherheit der medizinischen Certitudo zu suchen. Und in der Tat: «Ein philosophischer Arzt, wenn er im einzelnen Fall noch so gewissenhaft vorgeht, wird immer von einer vorsichtigen Unsicherheit, von einem vernünftigen Pyrrhonismus begleitet, so daß er bei einer Operation eher zurückhaltend bleiben, als zu weit gehen wird.» ${ }^{8}$ Abgesehen von seinen Hinweisen auf die alten Prinzipien von Hippokrates, Asklepiades und Celsus greift Verri sogar zurück auf Pyrrhon von Elis, den Begründer der skeptischen Schule, welcher lehrte, die allerhöchste Weisheit sei in Aphasie und Ataraxie zu suchen. Die Medizin verdient also die Bezeichnung (Vermutungswissenschaft〉. Verri - der zwar kein Mediziner ist - versteht letzten Endes die Medizin, oder besser die Klinik als die «Wissenschaft vom kranken Individuum». («Wissenschaft des Individuellen») ${ }^{9}$, wobei dieser Begriff eigentlich schon ein halbes Jahrhundert zuvor von Giacinto Viola, dem Kliniker für innere Medizin an der Universität Bologna, formuliert wurde, aber bestimmt nicht von all jenen angenommen wird, die sich streng an die Galileischen Prinzipien halten. Für die Vernunft ist also die Unsicherheit, Folge der Unkenntnis der «Prinzipien der Dinge», notwendigerweise mit der Annahme der einzig möglichen Lösung verbunden: mit dem Rückgriff auf die praktische Erfahrung ${ }^{10}$. Unvermeidlich ist hier der Verweis auf Hippokrates; unter anderem bezieht sich Verri auf die Thesen des Iatrophysikers Sanctorius Sanctorius, aber er betont, daß das Handeln des Arztes nur dann wirksam und möglich ist, wenn dank der hippokratischen Lehre die Erfahrung die Möglichkeit einer Hilfe verspricht.» ${ }^{11}$ Verris Position liegt in der Epoche nach Morgagni; trotzdem sieht er die Möglichkeit - wenn nicht das Erfordernis - das hippokratische Denken auf der klinischen Ebene positiv anzuwenden. Diese Haltung des lombardischen Denkers ist nicht verwunderlich; sie entspricht ganz der Orientierung der medizinischen Schule in Padua nach Morgagni ${ }^{12}$, sowie dem hippokratischen Purismus der Wiener Schule ${ }^{13}$.

Die Kultur der Aufklärung findet bei Morgagni ${ }^{14}$, Boerhaave ${ }^{15}$ und Frank ${ }^{16}$ einen starken Impuls zur Neuorientierung des medizinischen 
Studiums. Verris Vorstellungen sind folgende: Die Logik, das heißt «der Ausgangspunkt des menschlichen Wissens», die Kenntnis der lateinischen und der französischen Sprache, ferner die Statik, die Hydrostatik, die Geometrie, die Algebra, die Physik, die Botanik und die Chemie, - unentbehrliche Disziplinen, die jedoch einer geeigneten Dosierung bedürfen; schließlich die Anatomie, jedoch «erläutert und vergleichend» und nicht nur «unfruchtbare Nomenklatur» ${ }^{17}$ : das sind die wichtigsten Elemente des Unterrichts, entweder Haupt- oder Nebenfächer. «Die anatomischen Spitzfindigkeiten sind für den Mediziner von geringem Nutzen, sie sind Gegenstände, welche die Chirurgen und die Zeichner angehen.» ${ }^{18}$ Verris Stellungnahme ist zwar die eines Laien, sie kann aber vielleicht heute noch insofern akzeptiert werden, als man das Erfordernis einer besonderen Ausbildung des Internisten in Biochemie und des Chirurgen in Anatomie anerkennt.

Äußerst interessant und kritisch ist Verris Klassifikation der Ärzte; er unterscheidet diejenigen, die gute Ärzte werden wollen, und diejenigen, die bloß als guter Arzt gelten wollen ${ }^{19}$. Will einer den richtigen Weg einschlagen, - vorausgesetzt, daß er die oben erwähnten propädeutischen Kenntnisse erworben hat - so muß er die Lektüre ausgezeichneter Autoren pflegen, ihre Methoden prüfen, über Krankheitsverlauf, Symptome und Therapie nachdenken und «von der Erleuchtung und Erfahrung der Jahrhunderte Gebrauch machen». ${ }^{20}$ Wer sich aber damit begnügt, bloß im Ruf eines guten Arztes zu stehen, dem gibt Verri das ironische Rezept mit: «Gehen Sie in alle öffentlichen Schulen, lassen Sie sich zum Doktor machen, schließen Sie sich den Anhängern eines guten «Pulsisten〉 an, nutzen Sie viele Paar Schuhe ab, lernen Sie, wie man zwanzig Rezepte schreibt, lernen Sie vierzig griechische Wörter und dreißig Aphorismen von Hippokrates auswendig, preisen Sie die Verdienste der Pulslehre, denken Sie sich neue Wörter und Sätze aus und bereichern Sie damit ihren Wortschatz, stecken Sie getrost Ihr Honorar ein und vor allem beten Sie zum Himmel, daß die Fortschritte, die wir in der europäischen Philosophie jeden Tag verzeichnen, nicht andauern.» ${ }^{21}$ Der auserlesene Arzt muß «ein Mensch sein, der sich auf die eigene Kunst sehr wenig einbildet, ein Mensch, der seinen Beruf gründlich gelernt hat und dazu einen bescheidenen und ruhigen Charakter aufweist». ${ }^{22}$

Unter den klinischen Untersuchungsmethoden Galens waren die Beurteilung des Pulses und des Urins grundlegend. Diese beiden Verfahren - die, allerdings mit moderneren Verfahren kombiniert, noch in der heutigen Medizin Verwendung finden - standen in jener Medizin der Alten in hohem Ansehen, manchmal sogar als einzige Untersuchungsmethoden. Verri zeigt 
uns, daß die Schule der «Pulsisten» am Ende ausgeartet war. Es war sogar behauptet worden, man könne «aus der Pulsation der Arterie eine Unzahl von Krankheiten diagnostizieren». ${ }^{23}$ Verri läßtsich in die damalige Polemik ein, (hier könnten wir an die primären und sekundären Qualitäten Galileis, sowie an die Sanktorischen Kriterien für deren Beurteilung anknüpfen) er bezweifelt die Treffsicherheit bei der subjektiven Beurteilung unklarer Krankheitserscheinungen und fragt sich, «ob es möglich ist, durch bloßes Tasten (und ohne Uhr, welche die Sekunden und womöglich sogar die Zehntelssekunden anzeigt) winzige Unterschiede im Pulsschlag zu erkennen». ${ }^{24}$ Verri hält den Pulsisten für einen Betrüger, der bis zu 2000 Scudi monatlich verdient ... «mit einer Leistung, die für die Gesellschaft nutzlos, ja sogar gefährlich ist.» ${ }^{25}$ Für diesen übermäßigen Gewinn muß er allerdings wieder büßen, denn er lebt «in ständiger Angst, entlarvt zu werden, und muß sich endlos bemühen, die eigene Unwissenheit zu verhehlen, und muß jeder Gelegenheit ausweichen, einem richtigen Arzt zu begegnen». ${ }^{26}$

Es ist kaum nötig, darauf hinzuweisen, daß diese Auffassung des Berufsethos in Verbindung steht mit den aufgeklärten Humanitätsidealen, d.h. einer allgemeinen Menschenliebe - ohne Ansehung von Klassen und Traditionen - und mit einem Kampf gegen jeden Betrug. Den Prinzipien von Verri entspricht das Postulat, Verbreitung des Wissens sei eine moralische Pflicht der Philosophen und aller Wissenschaftler in ihrem Kampf gegen Scharlatanerie und jede Art von intellektueller Falschmünzerei.

Die praktische Erfahrung spielt in der Medizin eine wichtige Rolle, aber sie muß der Niederschlag der Erfahrung vieler Jahrhunderte und vieler Menschen sein, die sich in einem einzigen Mann verkörpert. Erfahrung im Umgang mit den Kranken ist für die richtige Bewertung der Krankheitssymptome erforderlich. Aber auch das durch Bücher vermittelte Wissen darf nicht geringgeschätzt werden. «Wer das Gegenteil behauptet» und Sie veranlaßt, sich nur praktisch zu üben, «der macht aus Ihnen höchstens einen guten Krankenwärter, aber keinen tüchtigen Arzt». ${ }^{27}$ Mit andern Worten: Bücherstudium und Beobachtung am Krankenbett sind die Schwerpunkte einer gründlichen Ausbildung. Wenn Verri hier - und nicht nur hier - «die Erfahrung vieler Jahrhunderte» verherrlicht, kann man sich fragen, ob er wirklich ein Mann der Aufklärung war, die doch als antihistorisch gilt. Aber dieser Antihistorismus war eher ein Antitraditionalismus, eine Ablehnung der übermächtigen Autorität der Tradition.

Verris Überlegungen zur Ätiopathogenese sensu lato zeigen ein etwas naiv vereinfachtes Bild der sozialen Kontraste: die Angehörigen der untern 
Volksschichten erkranken infolge zu schwerer Arbeit und mangelhafter Ernährung, die «Herren» wegen ihrer Unmäßigkeit. Krankheiten können beim Volk verhütet werden durch angemessene Ruhezeiten und gesunde, nahrhafte Kost, bei den Vornehmen durch Mäßigkeit, Körperbewegung und Zerstreuung ${ }^{28}$. Streng urteilt Verri über die Therapie, bei der man nur zu oft auf nicht rational begründete Anwendung von Medikamenten zurückgriff.

Er habe noch nie erlebt, daß ein todkranker Mensch durch Ärzte gerettet worden sei, daß chronische Kopfschmerzen auch durch Konsultation zahlreicher Ärzte geheilt wurden oder daß ein Zahnschmerz dank einem Heilmittel und ohne Zahnextraktion verschwunden sei ${ }^{29}$. Wie man sieht, ist Verris Haltung zu diesem Problem sehr skeptisch. Vielleicht liegt der Grund dieser Skepsis in persönlichen Erlebnissen, vielleicht auch im Versuch, durch diese besondere Strenge hinsichtlich therapeutischer Mißerfolge größere darstellerische Schärfe zu erzielen.

Die Chirurgie genießt bei Verri weit größere Sympathie und Vertrauen, vorausgesetzt allerdings, daß es sich dabei um eine von der Medizin unabhängige Chirurgie handelt, die «der Chirurg mit eigenen Händen ausübt». ${ }^{30}$ Diese Bemerkung «mit eigenen Händen» ist sehr interessant, weil sie an die Anschauungen von Leonardo und Vesal (15. und 16. Jahrhundert) über die Wichtigkeit des manuellen Handelns in jeder praktischen oder wissenschaftlichen Tätigkeit anknüpft, im Gegensatz zu den Methoden der scholastischen Medizin ${ }^{31}$. Diese Chirurgie besitzt ein sicheres Fundament, das auf der Anatomie fußt: Tatsächlich. «Wer die Anatomie beherrscht - und das muß ein Chirurg -, der kann ein Leben retten, indem er eine zerrissene Arterie vernäht; er kann einen Fremdkörper aus dem Leib entfernen, ohne Schäden zu setzen; er kann einem Patienten das Augenlicht wiedergeben, indem er eine undurchsichtig gewordene Linse entfernt: hier gibt es keinen Zweifel, daß die Kunst des Chirurgen auf sicheren Grundlagen aufbaut und bestimmt von Nutzen ist.» ${ }^{32}$ Doch auch in der Welt der Chirurgie herrscht viel Scharlatanerie: «Ich weiß von einem Mann, dem der Chirurg um jeden Preis die Testikel entfernen wollte, der aber dann genas, ohne kastriert zu werden.» ${ }^{33}$

Besondere Erwähnung verdient noch das, was Verri «über die Pockenimpfung» schreibt ${ }^{34}$, obwohl darüber ein besonderer Aufsatz erforderlich wäre. Auf diesen an historischen Hinweisen sehr reichen Seiten zitiert er unter andern auch den «berühmten Haller», welcher die Pockenimpfung mit dem Ausdruck «modus circassicus» bezeichnet und über die schweren Folgen dieser Krankheit berichtet ${ }^{35}$. Die Anregung zu diesem Artikel muß 
ausgesprochen in seinen aufklärerischen Tendenzen gesucht werden: «Ich freue mich, die unvoreingenommen gesuchte Wahrheit gefunden zu haben und mich dabei bemüht zu haben, sie in meiner Darstellung derart zu beschreiben, daß sie mit weniger Mühe und Zeitaufwand verstanden werden kann, als ich dazu gebraucht habe.» ${ }^{36}$

Auch auf medizinischer Ebene spiegelt Verris Denken die ethischen und sozialen Anschauungen des Intellektuellenkreises wider, der sich um die in Mailand zwischen 1764 und 1766 erschienene Zeitschrift ‘Il Caffề bildete. Diese Zeitschrift ist ein typisches Beispiel für das politische Engagement im Dienste des aufgeklärten Absolutismus der österreichischen Monarchie. Es wurden vielleicht etwas ehrgeizige Programme entworfen, die sowohl auf dem Gebiet der Hygiene als auch der sozialen und operativen Medizin an die Idee der Gemeinnützigkeit appellierten. Es galt, die Öffentlichkeit von Unwissenheit und Vorurteilen zu befreien und die Bedingungen für eine Erneuerung der Gesellschaft zu schaffen. Dies vermochte ohne Zweifel das Entstehen einer fortschrittlichen «Mentalität», eines soziokulturellen Milieus zu fördern, das der Entwicklung der modernen Medizin in Italien großen Nutzen brachte.

\section{Anmerkungen}

${ }^{1}$ E. H.Ackerknecht, Medizin und Aufklärung. In Schweiz. Med. Wschr. 89 (1959), S.20-22 (hier 20).

${ }^{2}$ Aschoff, Diepgen, Goerke, Kurze Übersichtstabelle zur Geschichte der Medizin. 7.Aufl., Berlin-Göttingen-Heidelberg 1960, S.26.

${ }^{3}$ Pietro Verri wurde am 12. Dezember 1728 in Mailand geboren und starb dort am 28. Juni 1797. Er studierte auch in Wien. Um die entarteten Sitten des Adels und die Vorurteile des Volks zu bekämpfen veröffentlichte er zahlreiche satyrische Almanache; 1764 gründete er die Zeitschrift Il Caffê, die vom Mai 1764 bis Juni 1766 erschien. Die Mitarbeiter dieser Zeitschrift, obwohl auf religiöser und politischer Ebene konservativ, befürworteten die Abschaffung der wirtschaftlichen Bindungen sowie der Privilegien. Aus dieser Zeitschrift wurden die hier angeführten Zitate entnommen.

${ }^{4}$ P. Verri, Memorie appartenenti alla vita e agli studi di Paolo Frisi. In «Scritti Vari di Pietro Verri ordinati da Giulio Carcano e preceduti da un Saggio civile sopra l'Autore per Vincenzo Salvagnoli», 2. Bd., Firenze 1854, S. 313.

${ }^{5}$ F. Diaz, Illuministi lombardi. In E. Cecchi und E. Sapegno, Storia della Letteratura Italiana, 6. Bd., Il Settecento, Milano 1968, S. 168.

${ }^{6}$ P. Verri, Sulla Medicina. (s. Anm. 4) S. 39.

7 Ebenda, S. 40.

${ }^{8}$ Ebenda, S. 41 . 
${ }^{9} \mathrm{G}$. Viola, La scienza a carattere universale e la clinica come scienza dell'individuale. In «Medicina interna» (hrsg. von A.Ceconi u. F. Micheli), 2.Aufl., 1.Bd., Torino 1936, S.16-19.

${ }^{10}$ P. Verri, Ricordi disinteressati e sinceri, De'Medici, (s. Anm.4) S. 234.

11 Ebenda, S. 235.

${ }^{12}$ Vgl. dazu: L. Premuda, Revival ippocratico a Padova in epoca post-morgagnana. In: Atti del XXIX Congresso nazionale di storia della medicina (a cura di E. Greco (Casale Monferrato 22.-23.-24. Settembre 1978). Casale Monferrato 1979, S.413-424.

${ }^{13}$ Vgl. dazu: E. Lesky, Die Wiener Medizinische Schule im 19. Jahrhundert, Graz - Köln 1965, S. 39-44.

${ }^{14}$ Vgl. dazu: Introduzione «Nova Institutionum Medicarum Idea» von G.B. Morgagni, Versione italiana e introduzione di Loris Premuda, Padova 1982.

${ }^{15}$ Siehe dazu: L.Premuda, La reazione metodologica di Hermann Boerhaave alla scienza cartesiana. In «Atti della V Biennale della Marca e dello Studio Firmano per lo Studio della Medicina», Fermo 1963, S.437-447.

Ders.: Beobachtungen und kritische Betrachtungen über die methodologische Grundlage von Hermann Boerhaave. In "Boerhaave and His Time", Papers read at the International Symposium in Commemoration of the trecentenary of Boerhaave's Birth (Leiden, 15.-16. November 1968) Leiden 1970, S.40-59.

${ }^{16}$ Vgl. dazu: E. Lesky, Johann Peter Frank als Organisator des medizinischen Unterrichts. In: Sudhoffs Archiv 39 (1955), S. 1-29.

${ }_{17}$ P. Verri, siehe Anm. 6, S. 42-43.

${ }^{18}$ Ebenda S. 43.

${ }^{19}$ Ebenda S. 38-39.

${ }^{20}$ Ebenda S. 43-44.

${ }^{21}$ Ebenda S. 48.

${ }^{22}$ P. Verri, siehe Anm. 10, S. 238.

${ }^{23}$ Ders., siehe Anm. 6, S. 46.

${ }^{24}$ Ebenda.

${ }^{25}$ P. Verri, Dei lucri de'medici. Siehe Anm. 4, S. 186.

${ }^{26}$ Ebenda, S. 187.

${ }^{27}$ P. Verri, siehe Anm. 6, S. 45.

${ }^{28}$ Ders., siehe Anm. 10, S. 232-233.

${ }^{29}$ Ebenda, S.232. Über die chaotische Situation der Therapie im 18. Jahrhundert lese man E. H. Ackerknecht, Therapie von den Primitiven bis zum 20. Jahrhundert. Stuttgart 1970, S. 78-94.

${ }^{30}$ P. Verri, Siehe Anm. 10, S. 238-239.

${ }^{31}$ Siehe dazu: L.Premuda, Sintesi di interessi artistici e scientifici nei disegni anatomici di Leonardo. In «Storia della iconografia anatomica», Mailand 1957, S.65-89;

Ders., Aspetti filosofici dell'opera vesaliana. In: Atti del XX. Congresso Nazionale di Storia della Medicina (Rom, 10.-11. Oktober 1964), Roma 1964, S.397-410;

Ders. Premessa. In Andrea Vesalio, Prefazione alla "Fabrica» e lettera a G.Oporino (Premessa, versione e testo a fronte a cura di L.Premuda), Padova 1964, S. 62.

${ }^{32}$ P. Verri. Siehe Anm. 10, S. 239.

${ }^{33}$ Ebenda, S. 240. 
${ }^{34}$ P. Verri, Sull'innesto del vaiuolo. Siehe Anm. 4, S.193-230.

${ }^{35}$ Ebenda, S. 197 u. 207.

${ }^{36}$ Ebenda, S. 230.

\section{Summary}

In this work the author considers some writings on medecine and physicians of Pietro Verri, the well-known philosopher, economist, politician and literate.

The arguments brought by the Milanese thinker reveal an interesting critic power and they suitably insert in the trend of illuministic medecine. Verri delays on the distinction betwen "a good physician" and who intends "to be vulgarly praised as a good physician".

Verri's critical reflexions reveal a sharp mind and a keen intellect and they are worth considering also at present. Some remarks on surgery and surgeon practice are of lesser interest. In all his prose we feel the ethic, scientific and methodologic trend of Illuministic age.

\section{Loris Premuda}

Professore all'Istituto di Storia della Medicina dell'Università di Padova

Via G. Falloppia 50

I-35100 Padova 